\title{
RECENT INNOVATIVE SOLUTIONS IN EDDY CURRENT NON-DESTRUCTIVE DIAGNOSIS
}

Recent innovative solutions in eddy current non-destructive evaluation of conductive materials are reported in the paper. Two main areas influencing reliability and preciseness of real cracks diagnosis are discussed. Advances in sensor technology are concerned at first. Innovative sensor design allowing sensing all the three spatial components of the perturbation electromagnetic field is presented. A novel approach for automatic diagnosis of real cracks from two-dimensional eddy current response signals is then introduced. The tabu search is applied for the three-dimensional reconstruction of partially conductive cracks.

Keywords: Non-destructive evaluation, eddy currents, sensors, diagnosis.

\section{Introduction}

Many structures require periodical inspection to keep safety, reliability as well as quality of various processes. It is especially crucial in areas where possible damages could lead to high economic and/or environmental losses, such as nuclear, petrochemical and transportation industries.

Recent trends in maintenance are embracing the so-called damage tolerance approach, wherein an element is actively used up to a certain point, beyond which the structural integrity of a structure could be affected. Replacement is thus performed at the end of the element service life, which helps in decreasing operating costs of the whole system, while keeping required level of safety, reliability and quality.

Modern approaches in the maintenance such as System Health Monitoring and Condition Based Maintenance come out from the damage tolerance approach. Degradation detection of a construction element must be accompanied by interpretation of the measured data in order to estimate the extent of the degradation and to predict its future development in consequence. The first two phases are inherently associated with Non-Destructive Evaluation (NDE) of materials. Enhancing NDE methods is therefore very important for accomplishing their challenging missions.

Different physical principles are utilised for the NDE of materials. Eddy current testing (ECT) is one of the widely utilized electromagnetic methods. It originates from the electromagnetic induction phenomena and the principle of ECT underlies in the interaction of induced eddy currents with structure of an examined body [1]. There are many advantages such as high sensitivity for surface breaking defects, high inspection speed, contact-less inspection, versatility, maturity of numerical means that account for continuously enlarging application area of the ECT even for biomaterials inspection [2]. However, in contrast to the simplicity of the method, recent trends in NDE open several challenging issues.

ECT is a relative method and the inverse problem is ill-posed [3]. Therefore, evaluating dimensions of a detected defect from ECT response signals can be quite difficult [4]. ECT instruments provide raw data with limited or absent capability of interpreting quantitatively the data [5]. Typically, evaluation relies on calibrated curves measured on pre-fabricated etalons and on the skills of an operator. Recently, the progress in powerful computers has allowed developing of automated procedures to make decisions. Quite satisfactory results are reported by several groups for automated evaluation of artificial slits [4] and even for several parallel notches [6]. However, evaluation of real cracks, especially stress corrosion cracking (SCC), from ECT response signals remains still very difficult. Many unsatisfactory results are reported when the automated procedures originally developed for non-conductive cracks are employed in the evaluation of SCCs. It is stated that one of the possible reasons is lack of sufficient information [4].

The authors have been working in the field of eddy current non-destructive evaluation for a long period. Their current activities are focused especially on enhancing information level of sensed data as well as on their correct and reliable interpretation. The paper addresses two important aspects in the light of the state-ofart. Advances in the sensor technology are discussed at first. Innovative solution of ECT probe providing possibility to detect all the three spatial components of the perturbation electromagnetic field is introduced in the next section. Interpretations of sensed data are

\footnotetext{
* Ladislav Janousek ${ }^{1}$, Mihai Iulian Rebican ${ }^{2}$, Milan Smetana ${ }^{1}$, Tatiana Strapacova ${ }^{1}$, Anton Duca ${ }^{2}$, Viera Pernisova ${ }^{1}$

${ }^{1}$ Department of Electromagnetic and Biomedical Engineering, Faculty of Electrical Engineering, University of Zilina, Slovakia,

E-mail: janousek@fel.uniza.sk

${ }^{2}$ Faculty of Electrical Engineering, University Politehnica of Bucharest, Romania
} 
analysed in consequence. A novel approach for diagnosis of real cracks from eddy current testing response signals is proposed. The tabu search is applied for the three-dimensional reconstruction of partially conductive cracks. Uniqueness of the proposal lies in the utilization of two-dimensional response signals for the three-dimensional inversion. Moreover, all the three spatial components of the perturbation electromagnetic field are taken as the response signals in order to tackle severe problem connected with diagnosis of partially conductive cracks.

\section{Advances in sensor technology}

ECT probes are one of the most important elements in NDE, because they transfer information between an ECT instrument and a conductive object through the induction coupling. An optimal ECT probe should assure [7]:

- high sensitivity to expected defects,

- high probability of detection of expected defects,

- possibility to distinguish parameters (location, dimensions, etc.) of expected defects.

Exciting coil(s) of ECT probe should thus induce eddy currents with high density and such distribution that the eddy current vector lines are significantly perturbed when a defect is in presence. The detection element should assure that maximum of the perturbation electromagnetic field is sensed.

An exciting system of ECT probes is usually made of inductance coils. Properties of the system depend on number, shape, configuration, orientation, dimensions and connections of exciting coils. Due to simplicity of analysis, synthesis, construction and production usually exciting coils of a circular shape or a rectangular one as shown in Fig. 1 are used to build up the ECT probes. They can be oriented normally (Fig. 1a) or tangentially (Fig. 1b) regarding the surface of a tested body. The shape and the orientation of exciting coil(s) determine distribution of the eddy current density vector in a tested body. The normal coils induce circular-like distribution of eddy currents and the tangential coils induce eddy currents with so called uniform distribution; however, the later exhibit directional properties.

There is one particular disadvantage of the ECT originating from its principle. Eddy currents are quite dense at a surface of a conductive material and they decay almost exponentially going inside the material due to the well known skin-effect. According to the electromagnetic field theory, the distribution of eddy currents along material depth depends mainly on a testing frequency and the electromagnetic parameters of a material as it is given by the well known equation for the standard depth of penetration:

$$
\delta=\frac{1}{\sqrt{\pi f \mu \sigma}},
$$

where $\delta[\mathrm{m}]$ is the standard depth of penetration, $f[\mathrm{~Hz}]$ is the inspection frequency, $\mu\left[\mathrm{H} \cdot \mathrm{m}^{-1}\right]$ and $\sigma\left[\mathrm{S} \cdot \mathrm{m}^{-1}\right]$ are the magnetic permeability and the electric conductivity of the inspected material, respectively. However, there are much more parameters with substantial influence on this distribution under real conditions, such as shape of coils, their dimensions, configuration of inspection, material thickness, etc.

Influence of the parameters defined above on the eddy current attenuation in a conductive material has been analyzed. The analyses have been carried out in order to optimize exciting coils for a new design of ECT probe with optimal features. There are especially two parameters of a circular exciting coil oriented normally
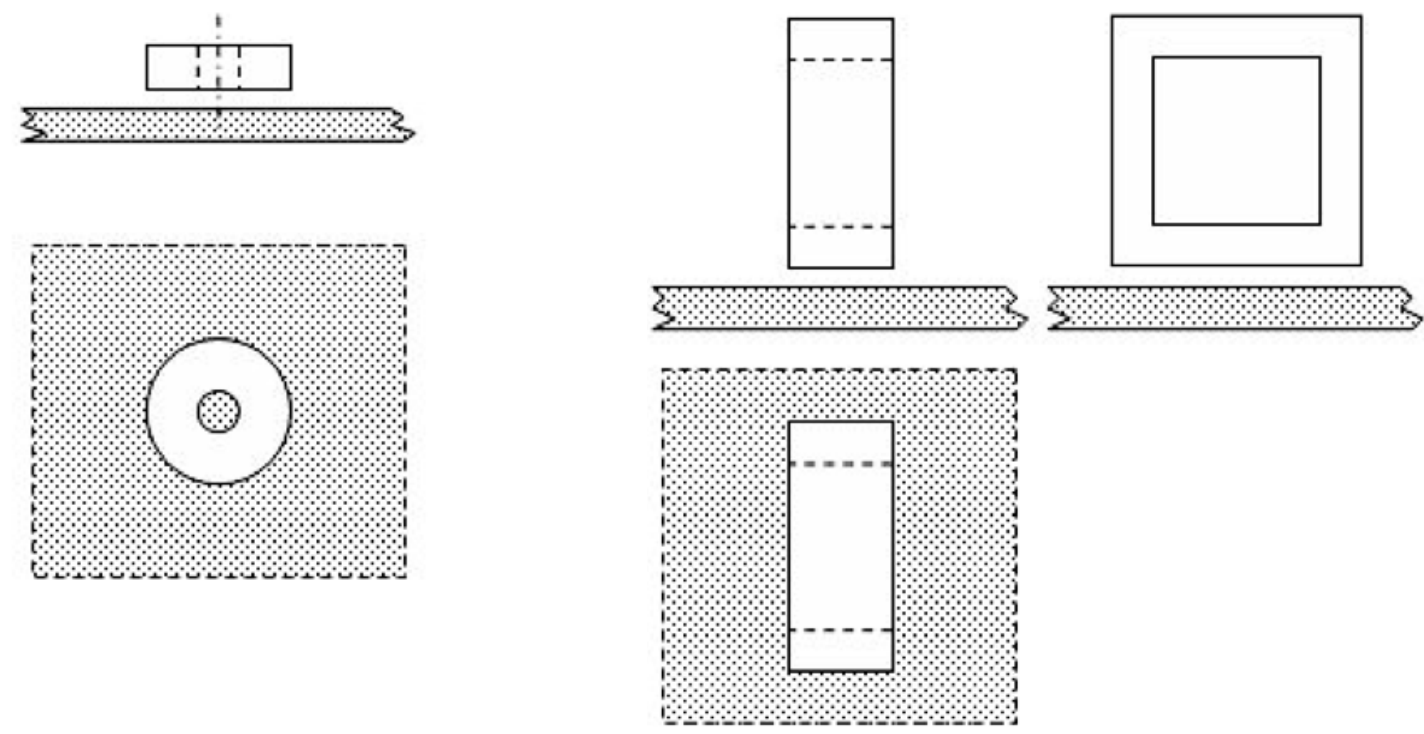

a) normally oriented circular coil

b) tangentially oriented rectangular coil

Fig. 1 Basic shapes and orientations of exciting coils 
regarding a material surface (see Fig. 1a) that substantially influence the eddy current attenuation in the material: 1) outer radius of the coil; 2) clearance between the coil and the material surface, so called lift-off [8].

Particular results of the investigations are shown in Fig. 2 for a plate made of SUS316L with a thickness of $10 \mathrm{~mm}$ and the electromagnetic parameters $\sigma=1.35 \cdot 106 \mathrm{~S} \cdot \mathrm{m}^{-1} \mu_{r}=1$. Cross section of the coil winding is adjusted to $1 \times 1 \mathrm{~mm}^{2}$. Attenuations of the eddy current density absolute value along the material depth for coils with different radius are shown in Fig. 2a. The dependences are shown in normalized values for the frequency of $f=1 \mathrm{kHz}$ and the lift-off $l f=0.5 \mathrm{~mm}$. Black dotted line denoted as "theory" is calculated according to:

$$
J_{e z}=J_{e s} e^{-\frac{z}{\delta}}
$$

where $J_{e z}\left[\mathrm{~A} \cdot \mathrm{m}^{-2}\right]$ is the absolute value of eddy current density at distance $\mathrm{z}[\mathrm{m}]$ from boundary between the air and the conductive material and $J_{e s}\left[\mathrm{~A} \cdot \mathrm{m}^{-2}\right]$ is the absolute value of eddy current density at the material surface, $\delta[\mathrm{m}]$ is the standard penetration depth given by (1). Influence of the lift-off on the eddy current profile along the material depth direction for the same frequency is shown in Fig. 2b. It is obvious from the presented results that the attenuation of eddy current density strongly depends on the coil diameter as well as on the lift-off. However, these influences gradually vanish with increasing ratio between the plate thickness and the standard depth of penetration. It should be noted that larger coils and/or larger lift-offs provide deeper penetration of eddy currents inside a material and thus it would provide better resolution. However, sensitivity can be decreased. Sophisticated design of the exciting system requires therefore appropriate compromise to reach essential performance of an ECT probe.

Detection elements of conventional ECT probes are usually made of inductance coils. However, recently, magnetic sensors based on either Hall effect, Anisotropic-Magneto-Resistance (AMR), Giant-Magneto-Resistance (GMR) effect, or Fluxgate magnetometers have been successfully used for crack detection in ECT [9].
The most common Fluxgate sensor consists of two coils wrapped around the high-permeability ferromagnetic core. Magnetic induction of the core is changed by the presence of an external magnetic field. A driving signal is applied to one of the coils and the measured signal is taken from the second one. Changes in core permeability affect the measured signal as its amplitude variations.

AMR sensors usually consist of four ferromagnetic resistor stripes connected in Wheatstone bridge. Changes of magnetic resistance due to applied magnetic field can be up to 3\%. AMR sensors offer small size and noise sensitivity.

GMR sensors use the phenomenon of large magnetic field dependent changes in resistance in thin ferromagnetic/nonmagnetic metallic multilayer structures. Comparing to small changes of resistance in AMR, GMR material can achieve about 10\%-20\% changes in resistance. The resistance of two thin ferromagnetic layers separated by a thin nonmagnetic conducting layer can be altered by changing the moments of the ferromagnetic layers from parallel to anti-parallel. Layers with parallel magnetic moments will have less scattering at the interfaces, longer mean free paths, and lower resistance. Layers with anti-parallel magnetic moments will have more scattering at the interfaces, shorter mean free paths, and higher resistance.

Among these, the magneto-resistive (MR) sensors offer a good trade-off in terms of performance versus cost. They have small dimensions, high sensitivity over a broad range of frequency (from hertz to megahertz domains), low noise; they operate at room temperature, and are inexpensive. It has been demonstrated that ECT probes with MR sensors perform better than the ones with inductance sensing coils for low-frequency applications, e.g. when detecting deeply buried flaws. This is because the electromagnetic sensors are sensitive to the magnitude of the magnetic field. In the case of inductive-based probes, the output voltage is proportional to the time variation of the magnetic field; therefore, their sensitivity is reduced at low frequencies.

The authors have analyzed properties of various sensors in order to design a novel ECT probe [10], [11]. Especially, perfor-

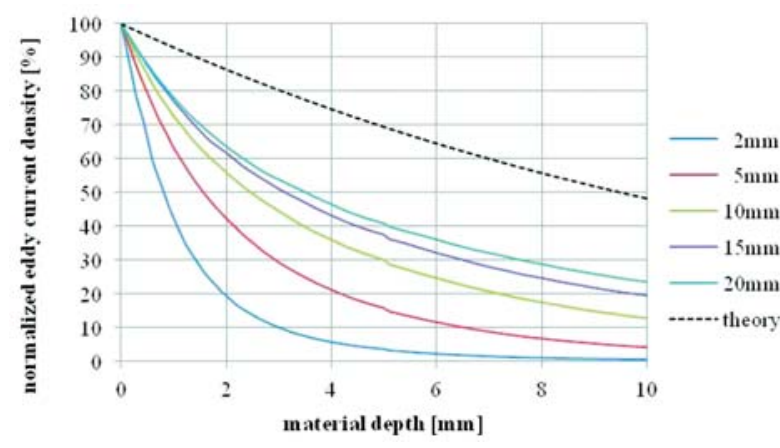

a) influence of the coil radius, If $=0.5 \mathrm{~mm}$

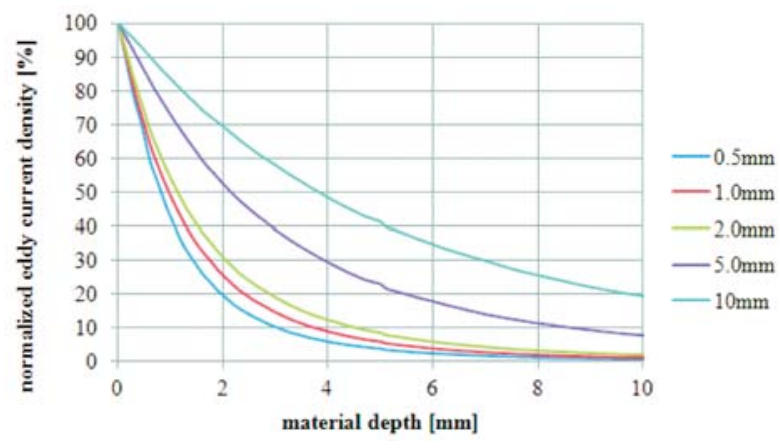

b) influence of the lift-off, $r_{c}=2 \mathrm{~mm}$

Fig. 2 Attenuation of eddy current density in normalized values along material depth, $f=1 \mathrm{kHz}$ 


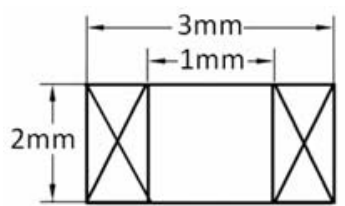

a) inductance coil

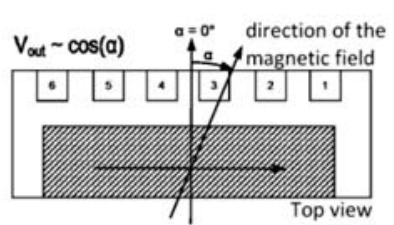

b) GMR sensor (Sensitec)

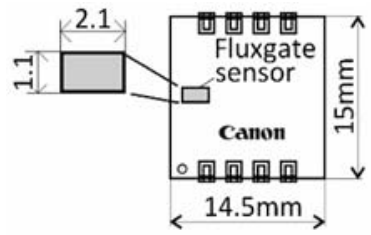

c) Fluxgate sensor

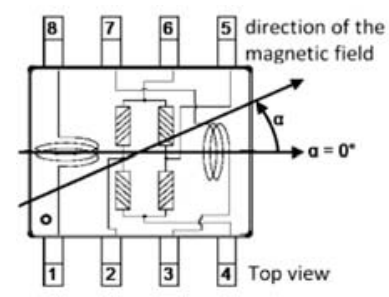

d) AMR Sensor (Sensitec)

Fig. 3 Sensors layout

mances of GMR, AMR and Fluxgate magnetic sensors and of an inductance coil, shown in Fig. 3, were compared under the same conditions based on numerical simulation as well as experimental measurements.

The results presented in [10], [11] proved that the magnetic sensors provide very similar resolution comparing to the inductance coils; however, they offer much better sensitivity especially for low frequency applications.

Common eddy current probes are designed in such a way that they sense only one component of the perturbation electromagnetic field. Usually, the most significant component is used for the evaluation. However, curved paths of eddy currents provide more information in principle.

Several studies of authors focused on enhancing information level of eddy current testing signals [12], [13]. The original idea is based on sensing of all three spatial components of the perturbation electromagnetic field. The studies have revealed that the uncertainty in cracks' evaluation is reduced when the enriched information is utilized for the purpose.

A novel ECT probe has been designed accounting for increased information level of response signals according to the previous works shortly introduced above. Layout of the probe together with its photo is shown in Fig. 4.
The probe consists of two circular exciting coils positioned apart from each other and oriented normally regarding the surface of an inspected material. The circular coils are connected in series but magnetically opposite to induce uniformly distributed eddy currents in the specimen. A detection system of the probe is composed of three GMR sensors oriented along three axes perpendicularly to each other. The detection system is located in the centre between the exciting coils to gain high sensitivity as the direct coupling between the exciting coils and the sensors is minimal at this position. The following section presents innovative solution for automatic diagnosis of real crack employing the new probe.

\section{Advances in automatic diagnosis}

The progress in powerful computers has allowed developing of automated procedures to provide diagnosis of cracks from ECT response signals. Two approaches are utilized for the purpose in general: 1) deterministic and 2) stochastic [4], [6].

Usually, one dimensional signal gained by scanning just above an indicated crack along its length is taken as an input to the evaluation procedure. Mostly, three variables of the defect are estimated, its depth, length and position of its centre, while a profile, a width and the electromagnetic parameters of the defect are adjusted in advance. The deterministic methods are the model based. They

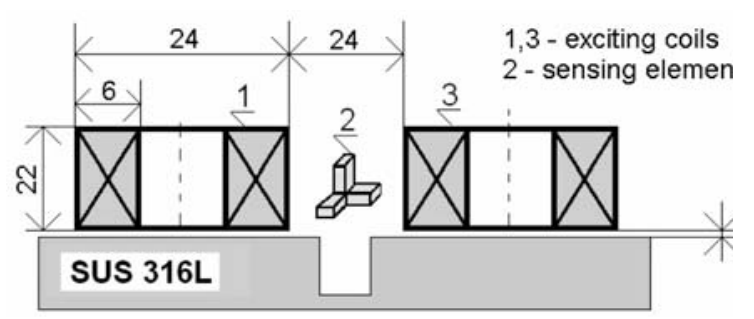

a) layout and dimensions

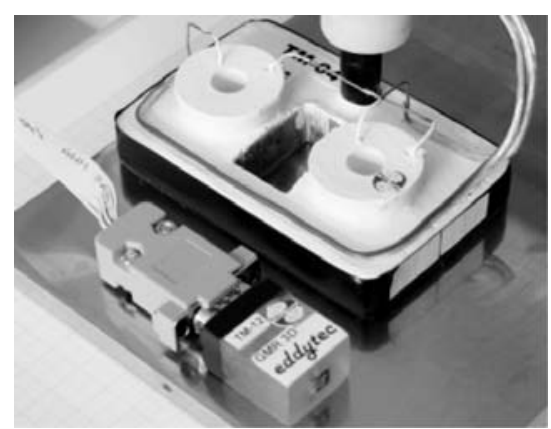

b) photo

Fig. 4 Novel ECT probe 
work according to the difference minimization between measured and simulated signals. The process is iterative and therefore large number of forward simulations is required. The stochastic approaches simulate the mapping between eddy currents signals and defect profiles based on many known datasets. So called evolution algorithms, for example neural networks, genetic algorithms, are utilized for the inversion.

The authors have already developed an algorithm for reconstruction of multiple slits from ECT response signals by means of a stochastic optimization method, such as tabu search [6]. The reconstruction of multiple slits was done in three-dimensions. Therefore, the scheme is also appropriate for reconstruction of a partially conductive crack, when its width as well as its partial conductivity have to be considered as variable.

This section presents a novel approach for automatic diagnosis of real cracks proposed by the authors using the new probe presented above. The tabu search is applied for the three-dimensional reconstruction of partially conductive cracks. Uniqueness of the proposal lies in the utilization of two-dimensional response signals for the three-dimensional inversion. Moreover, all the three spatial components of the perturbation electromagnetic field are taken as the response signals in order to tackle severe problem connected with diagnosis of partially conductive cracks. Effectiveness of the proposal has been studied by numerical means at first and particular results are presented here.

A plate specimen having the electromagnetic parameters of a stainless steel SUS316L is inspected here. The specimen has a thickness of $10 \mathrm{~mm}$, a conductivity of $\sigma=1.35 \mathrm{MS} / \mathrm{m}$ and a relative permeability of $\mu_{r}=1$. A single surface breaking crack appears in the plate. It is modelled as a structure having different electromagnetic properties from the base material. Configuration of the plate (region $\Omega_{0}$ ) with the crack (region $\Omega_{1}$ ) is shown in Fig. 5 . The crack region $\Omega_{1}\left(22 \times 2 \times 10 \mathrm{~mm}^{3}\right)$ is uniformly divided into a grid composed from $n_{x} \times n_{y} \times n_{z}(11 \times 5 \times 10)$ cells defining a possible crack geometry. The dimensions of each cell are $2.0 \times$ $\times 0.4 \times 1.0 \mathrm{~mm}^{3}$

The new eddy-current probe, shown in Fig. 4, is employed for the near-side inspection of the plate. Two-dimensional scanning, so called C-scan, is performed over the cracked surface with a liftoff of $l_{f}=1 \mathrm{~mm}$. The real and the imaginary parts of the output

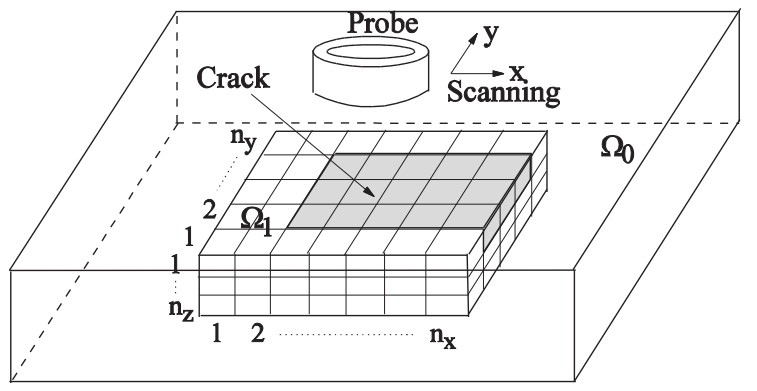

Fig. 5 Configuration of plate specimen with crack region absolute value [uT]

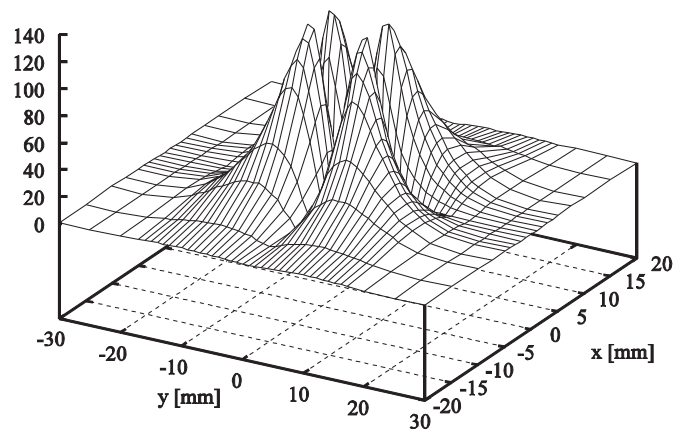

a) x-component $B_{x}$

absolute value [uT]

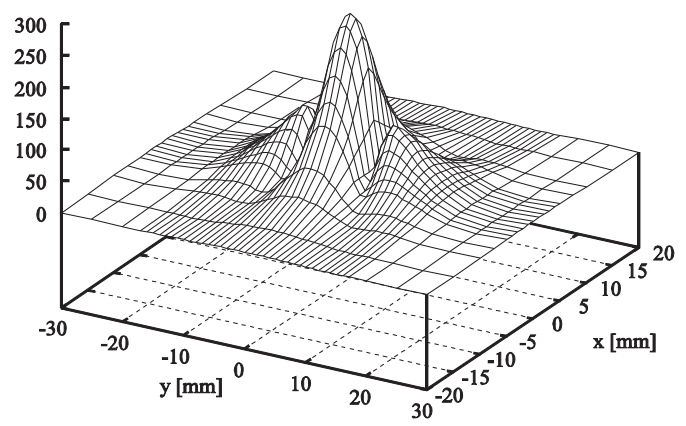

b) y-component $B_{y}$

absolute value [uT]

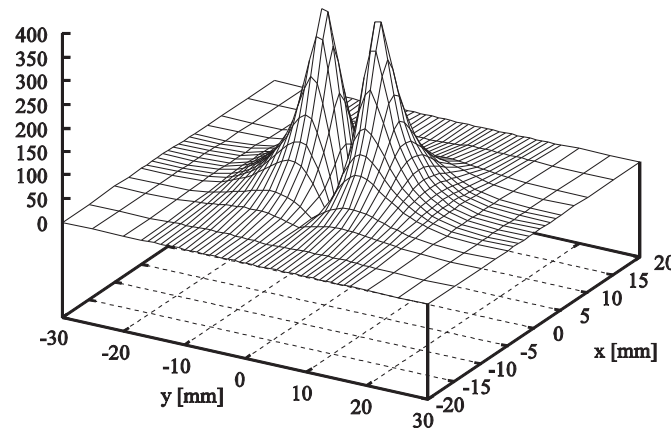

c) z-component $B_{z}$

Fig. 6 Absolute values of the sensed magnetic flux density vector $B$ vs. probe position, $l_{c}=10 \mathrm{~mm}, d_{c}=5 \mathrm{~mm}, w_{c}=0.2 \mathrm{~mm}, \sigma_{c}=0 \%$ of $\sigma$

voltages from all three GMR sensors corresponding to three spatial components of the perturbation electromagnetic field are sensed and recorded during the inspection. Example of crack signatures is shown in Fig. 6. Dependences of the three spatial components of the perturbation magnetic flux density vector on the probe position are displayed. The signals correspond to a crack of the cuboid 
shape with a length of $l_{c}=10 \mathrm{~mm}$, a depth of $d_{c}=5 \mathrm{~mm}$, a width of $w_{c}=0.2 \mathrm{~mm}$ and its partial conductivity is adjusted to $\sigma_{c}=0 \%$ of the base material conductivity $\sigma$. The crack is oriented along the $\mathrm{y}$-direction in the $10 \mathrm{~mm}$ thick SUS316L plate.

A database approach for fast-forward computation of the ECT response signals due to multiple cracks is used in this paper [6] The database is designed for a three-dimensional defect region and not as usually for a two-dimensional one where a crack width is considered as fixed. Thus, the ECT response signals can be simulated also for partially conductive cracks with variable width using the same database generated in advance. The area of the simulated two-dimensional ECT signals has surface dimensions of $100 \times 28$ $\mathrm{mm}^{2}$. The number of scanning points in the two directions is 50 and 70 , respectively.

Tabu search is employed for the three-dimensional diagnosis of a detected crack [6]. Three-dimensional model of the crack is shown in Fig. 7. The crack depth is considered as variable along the crack length. The crack parameter vector c consists of $n_{x}+3$ integers, $c=\left[i z_{1}, i z_{2}, \ldots, i z_{n x}, i y_{1}, i y_{2}, s\right]$, where $i z_{k}, k=1, n_{x}$ is the number of cells of the crack along the crack depth, $i y_{1}$ and $i y_{2}$ are the indices of the first and last cells of the crack along the width direction, and the crack partial conductivity is expressed as $\sigma_{c}=\mathrm{s} \%$ of the base material conductivity $\sigma$.

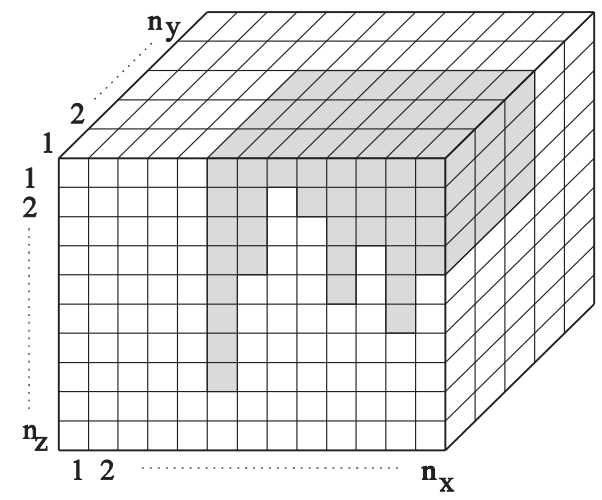

Fig. 7 Three-dimensional model of partially conductive crack

Figure 8 shows results of three-dimensional diagnosis of two partially conductive cracks when the crack model shown in Fig. 7 is employed for the inversion. In this case two profiles of a crack are considered. The first crack has the cuboid shape and its true parameters are as follows: length $l_{c}=10 \mathrm{~mm}$, width $w_{c}=1.2 \mathrm{~mm}$, depth $d_{c}=7 \mathrm{~mm}$ and partial conductivity $\sigma_{c}=8 \%$ of the material base conductivity $\sigma$. The result of reconstruction is shown in Fig. $8 \mathrm{a})$. As it can be observed the crack is precisely localized and also its length and width are exactly estimated. The depth profile does not perfectly copy the true one. However, the maximum depth is accurately assessed. A crack with elliptical profile is also reconstructed. The crack opening has a value of $w_{c}=0.4 \mathrm{~mm}$, its surface length is $l_{c}=14 \mathrm{~mm}$, the maximum depth is $d_{c}=4 \mathrm{~mm}$ and the crack's partial conductivity is adjusted to $\sigma_{c}=8 \%$ of the base material conductivity $\sigma$. The reconstruction result is shown in Fig. 8b). The crack width and its surface length are accurately assessed. The estimated crack position is minimally shifted $(0.4 \mathrm{~mm})$ in the crack width direction comparing the true position. The maximum depth is slightly overestimated of $1 \mathrm{~mm}$.

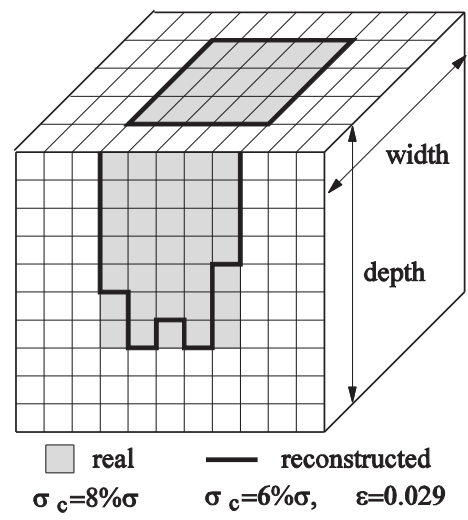

a) cuboid shape of crack

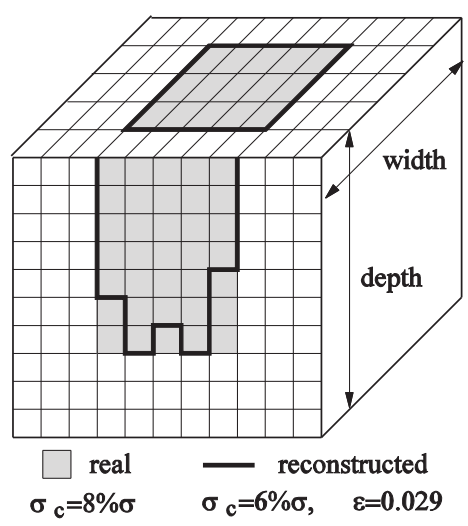

b) elliptical shape of crack

Fig. 8 Results of partially conductive cracks' reconstruction

The presented results proved effectiveness of the proposed novel approach of three-dimensional diagnosis of partially conductive cracks. ECT response signals gained during $\mathrm{C}$-scan together with acquiring all three spatial components of the perturbation electromagnetic field significantly improve the preciseness of inversion process using tabu search stochastic method.

\section{Conclusion}

The paper focused on recent $R \& D$ activities in the field of eddy current non-destructive evaluation of conductive materials. Two innovative solutions providing more reliable diagnosis of real cracks from eddy current response signals were presented. A new design of eddy current probe allowing sensing three spatial com- 
ponents of the perturbation electromagnetic field due to a crack was introduced. A novel approach for three-dimensional diagnosis of partially conductive cracks was proposed consequently. The tabu search stochastic method was employed for the reconstruction from eddy current response signals gained during two-dimensional scan of the probe. The presented results proved that the proposed approach allows quite precisely reconstructing three-dimensional profile of a partially conductive crack even with elliptical shape together with its partial conductivity.

Further work of the authors will concern especially experimental verifications.

\section{Acknowledgement}

This work was supported by the Slovak Research and Development Agency under the contracts No. APVV-0349-10 and APVV-0194-07.

This work was also supported by grants of the Slovak Grant Agency VEGA, projects No. 1/0765/11, 1/0927/11

This work has been co-funded by the Sectoral Operational Programme Human Resources Development 2007-2013 of the Romanian Ministry of Labour, Family and Social Protection through the Financial Agreement POSDRU/89/1.5/S/62557.

\section{References}

[1] JANOUSEK, L., T. MAREK, T., D. GOMBARSKA, D.: Eddy Current Non-destructive Evaluation of Conductive Materials. Communications - Scientific Letters of the University of Zilina, 1, pp. 29-33, 2006.

[2] STRAPACOVA, T., CAPOVA, K., JANOUSEK, L.: Artificial Heart valve Inspection Using Eddy Current Techniques. Communications - Scientific Letters of the University of Zilina, vol. 13, No. 2A, pp. 83-86, 2011.

[3] YUSA, N., HUANG, H., MIYA, K.: Numerical Evaluation of the Ill-posedness of Eddy Current Problems to Size Real Cracks. NDT\&E International, vol. 40, pp. 185-191, 2007.

[4] YUSA, N.: Development of Computational Inversion Techniques to Size Cracks from Eddy Current Signals. Nondestructive Testing and Evaluation, vol. 24, pp. 39-52, 2009.

[5] RUBINACCI, G., TAMBURINO, A., VENTRE, S.: Fast Numerical Techniques for Electromagnetic Nondestructive Evaluation. Nondestructive Testing and Evaluation, vol. 24, pp. 165-194, 2009.

[6] REBICAN, M., CHEN, Z., YUSA, N., JANOUSEK, L., MIYA, K.: Shape Reconstruction of Multiple Cracks from ECT Signals by Means of a Stochastic Method. IEEE Transactions on Magnetics, vol. 42, pp. 1079-1082, 2006.

[7] CHEN, Z., MITA, K.: A New Approach for Optimal Design of Eddy Current Testing Probes. J. of Nondestructive Evaluation, vol. 17, pp. 105-116, 1998.

[8] JANOUSEK, L.: Impact of Selected Parameters on Eddy Current Attenuation in Conductive Materials. Proc. of $9^{\text {th }}$ intern. conference ELEKTRO 2012, Rajecke Teplice, Slovakia, pp. 419-422, 2012.

[9] CAPOVA, K., CAP, I., JANOUSEK, L., SMETANA, M.: Recent Trends in Electromagnetic Non-destructive Sensing. Advances in Electrical and Electronic Engineering, vol. 7, pp. 322-325, 2008.

[10] SMETANA, M., JANOUSEK, L.: Evaluation of Inductance Coil and Fluxgate Magnetometer Under Harmonic and Pulsed Excitations in ECT. Intern. J. of Applied Electromagnetics and Mechanics, vol. 39, pp. 277-282, 2012.

[11] STRAPACOVA, T., JANOUSEK, L., SMETANA, M., CAPOVA, K.: Evaluation of Advanced Sensor Types Under Harmonic Excitation in ECT. The $17^{\text {th }}$ Intern. Workshop on Electromagnetic Nondestructive Evaluation: Proceedings, Rio de Janeiro - Brazil, pp. 77-78, 2012.

[12] JANOUSEK, L., SMETANA, M., CAPOVA, K.: Enhancing Information Level in Eddy-current Non-destructive Inspection. Intern. J. of Applied Electromagnetics and Mechanics, vol. 33, pp. 1149-1155, 2010.

[13] JANOUSEK, L., SMETANA, M.: Uncertainty in Depth Evaluation of Partially Conductive Cracks from Eddy Current Testing Signals. Communications - Scientific Letters of the University of Zilina, vol. 13, No. 2A, 2011, pp. 55-60, 2011. 